\title{
Simple Model of an Inductive Output Tube
}

\author{
Richard G Carter \\ Engineering Department \\ Lancaster University \\ Lancaster, LA1 4YR, UK
}

\begin{abstract}
This paper describes a simple method for modeling the performance of an Inductive Output Tube (IOT). The model reproduces the characteristics of an IOT with good accuracy over the full dynamic range of the tube.
\end{abstract}

Keywords: Microwave tube; Inductive Output Tube; IOT; Computer modelling.

\section{Introduction}

The Inductive Output Tube (IOT), illustrated in fig.1, was first proposed many years ago [1] and successful devices have since been developed for digital television broadcasting and for powering particle accelerators [2]. Although this type of tube is well-known a simple theory of its operation has not been presented in the literature as far as the author is aware.

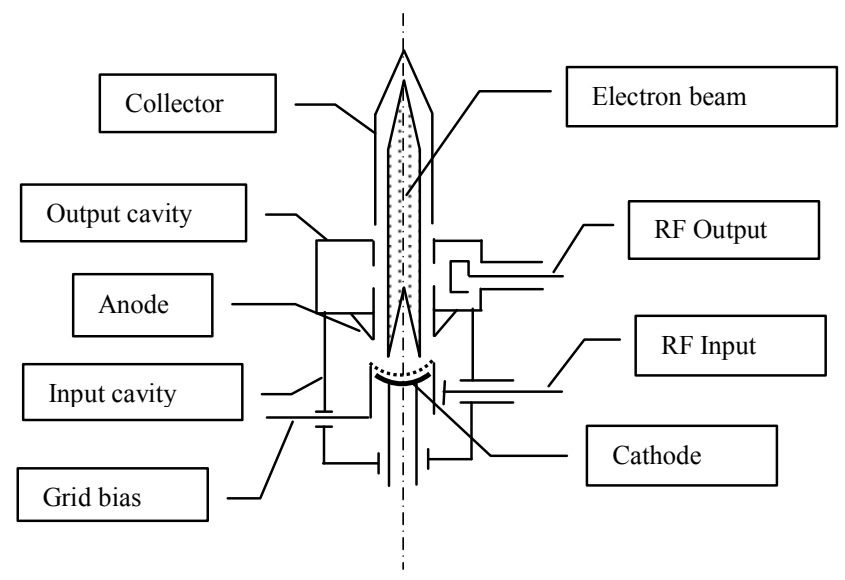

Figure 1. Arrangement of an Inductive Output Tube

\section{Theory}

The input stage of an IOT is the triode electron gun in which the current is determined by space-charge limited flow in the region between the cathode and the grid. It is generally agreed that the current-voltage relationship for a triode can be represented by [3]

$I=K\left(V_{g}+\frac{V_{a}}{\mu}\right)^{n}$

where $\mathrm{V}_{\mathrm{g}}$ and $\mathrm{V}_{\mathrm{a}}$ are the potentials of the grid and the anode with respect to the cathode and $K, \mu$ and $n$ are constants determined by the geometry of the triode. The value of $n$ is typically in the range 1.5 to 2.5 because the geometry of the space-charge limited flow depends upon the applied voltages as well as upon the arrangement of the electrodes. The current is zero when the term in the brackets is negative. In an IOT the grid voltage is given by

$V_{g}=V_{g 0}+V_{g 1} \sin \omega t$

where $V_{g 0}$ is the DC grid bias and $V_{g 1}$ is the amplitude of the RF input voltage applied to the grid. $V_{g 0}$ is commonly chosen so that the electron flow is just cut-off in the absence of RF input so that the triode is approximately in class B operation. By substituting for $V_{g}$ in (1) using (2) the beam current is obtained as a function of time once the values of the constants have been specified. The current can be expanded as a Fourier series so that

$$
I=I_{0}+I_{1} \sin \omega t+e t c \text {. }
$$

The DC and RF input powers are then

$P_{0}=I_{0} V_{a}$

and

$$
P_{1}=\frac{1}{2} I_{1} V_{g 1}
$$

If the RF impedance of the output gap is $R_{2}$ then the amplitude of the effective output gap voltage acting on the beam is

$$
V_{2 e f f}=M_{\text {eff }}^{2} I_{1} R_{2}
$$

where $M_{\text {eff }}$ is the effective gap coupling factor. It is wellknown that, when the gap voltage is small, and the electron velocity is effectively constant through the gap, the coupling factor is given by

$$
M=\frac{2 I_{1}(\gamma b)}{\gamma b \cdot I_{0}(\gamma b)} \frac{\sin \left(\beta_{e} g / 2\right)}{\left(\beta_{e} g / 2\right)}
$$

where $a$ and $b$ are the radius of the drift tube and the electron beam, $g$ is the length of the interaction gap, $I_{0}$ and $I_{1}$ are modified Bessel functions, $\beta_{e}$ is the ratio of the angular frequency $\omega$ to the electron velocity, and

$$
\gamma=\sqrt{\beta_{e}^{2}-(\omega / c)^{2}}
$$

In practice there is little difference between $\gamma$ and $\beta_{e}$ at the anode voltages typically used in IOT's and $\beta_{e}$ may be substituted for $\gamma$ in (7) without serious error. Because the electron velocity is not constant within the output gap it is assumed that, to a first approximation, the effective gap 
coupling factor $\left(M_{\text {eff }}\right)$ can be calculated setting $\beta_{e}=\frac{2 \omega}{u_{0}+u_{s}}$

in (7), where $u_{0}$ is the DC beam velocity and $u_{s}$ is the minimum spent-beam velocity given by

$u_{s}=c\left[1-\frac{1}{\left[1+\frac{\eta\left(V_{a}-V_{e f f}\right)}{c^{2}}\right]^{2}}\right]^{\frac{1}{2}}$

where $\eta$ is the charge to mass ratio of the electron. Since $V_{\text {2eff }}$ depends upon $u_{s}$ an iterative calculation is used to find mutually consistent values. When cavity losses are ignored the RF output power is

$$
P_{2}=\frac{1}{2}\left(M_{e f f} I_{1}\right)^{2} R_{2}
$$

so that the electronic efficiency is

$$
\eta_{e}=P_{2} / P_{0}
$$

It is shown in the following section that this gives a good approximation to the behaviour of an IOT if the value of $R_{2}$ is used as a variable parameter.

\section{Example}

The results of detailed measurements on an IOT are given in [4] and it is possible find a self consistent set of parameters which model this tube successfully. Table 1 in [3] gives sets of values of: beam current; input power; output power; efficiency and gain when $V_{g 0}=-105 \mathrm{~V}$. It was assumed that the beam current obeyed (1) and equations (3) and (5) were used to compute pairs of values of the DC beam current and the RF input power. Then $K, \mu$ and $n$ were adjusted to obtain a good fit to the experimental data. Figure 2 shows the comparison between the data and the simulation with $K=33 \times 10^{-6}, \mu=$ 150 and $n=1.5$.

The method described in the previous section was used to find the spent beam velocity. In the absence of full data it was assumed that $\beta_{e} a=1.0$ (calculated at the DC beam velocity), $b=0.6 a$ and $g=a$. The value of $R_{2}(26 \mathrm{k} \Omega)$ was adjusted to obtain the best fit to the experimental data for the transfer curve as shown in fig.3. The curves of gain and efficiency against input power can be computed in a similar manner. Figure 3 also shows transfer curves computed for other values of the grid bias voltage.

\section{Conclusion}

The method described provides a quick and easy way of modeling the performance of an IOT over its full dynamic range. It lends itself readily to parametric studies of IOTs to optimize their performance. The method of modeling the interaction between the electron bunches and the output cavity could also be applied to klystrons.

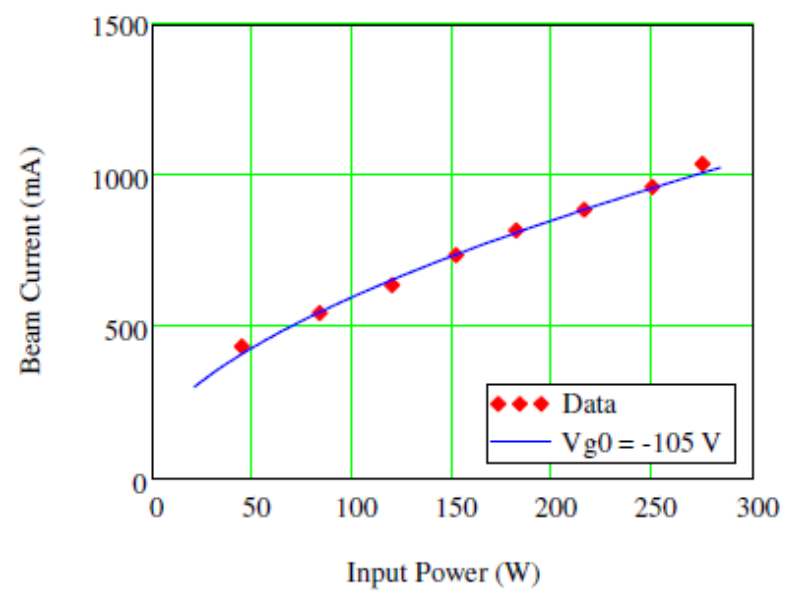

Figure 2. DC beam current vs. RF input power

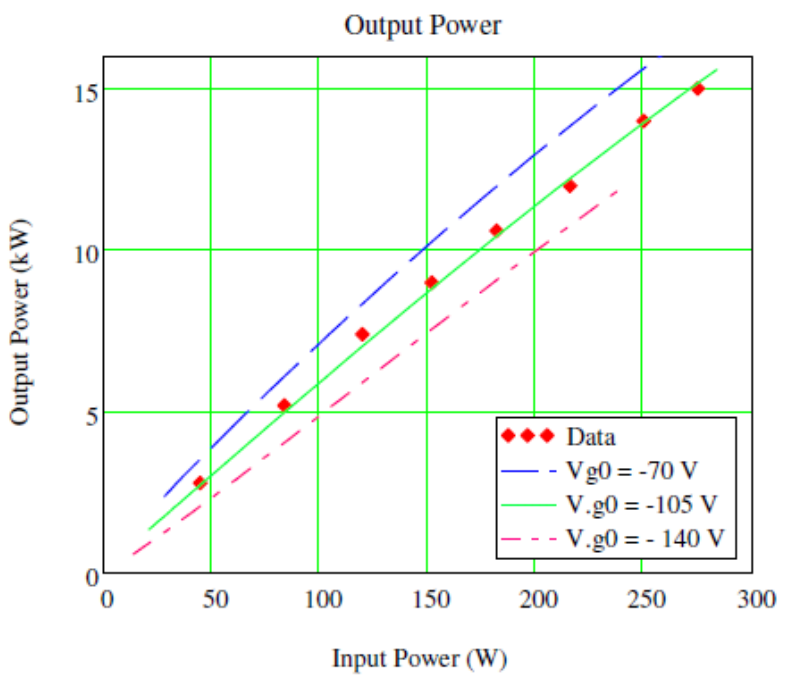

Figure 3. RF output power vs. RF input power

\section{References}

1. D.H. Preist and M.B. Shrader, "The Klystrode - an unusual transmitting tube with potential for UHF-TV", Proc. IEEE, vol.70, no.11, pp.1318-25 (1982).

2. H. Bohlen, Y. Li and R. Tornoe, "IOT RF power sources for pulsed and cw linacs", Proc. LINAC 2004, Lübeck, Germany, pp.574-77.

3. J.W. Gewartowski and H.A. Watson, Principles of Electron Tubes, D. van Nostrand (1965)

4. J.F. Orrett, A.J. Moss, P. Corlett and S. Buckley, "IOT testing at the ERLP", Proc. EPAC 2006, Edinburgh, Scotland, pp.1382-84. 